\title{
ANALYSIS OF A NOVEL NOZZLE USED FOR PULSE JET FILTRATION USING CFD SIMULATION METHOD
}

\author{
Li, H. X. ; Li, B. ; Choi, J. ; ${ }^{* *}$ Heo, J. ${ }^{* *} \&$ Kim, I. ${ }^{* *}$ \\ *School of Mechanical and Power Engineering, Henan Polytechnic University, Jiaozuo, 454000, China \\ ** Department of Chemical Engineering, Gyeongsang National University, Jinju, 660-701, South Korea \\ E-Mail: jhchoi@gnu.ac.kr
}

\begin{abstract}
The nozzle is one of the most important components of pulse jet systems. The optimization of a pulse jet system and its optimal operation condition are barely known because of the complicated turbulent mixing, compressibility effects, and even flow unsteadiness, which are generated in the nozzle system. In this study, a three-dimensional numerical model of a pulse jet system was established to analyse its operation characteristics by using computational fluid dynamics (CFD). The CFD simulation results were validated with existing experimental data. The flow dynamics of the pulse jet gas around the cleaning nozzle was numerically explored to predict the effects of nozzle shape and dimensions on the pulse cleaning performance in terms of pressure distribution, velocity distribution, primary mass, secondary mass, and entrainment ratio. The influences of the convergent ratio in the narrow $\left(R_{c h}\right)$ and wide $\left(R_{c w}\right)$ directions, as well as that of the divergent ratio $\left(R_{d w}\right)$ in the wide direction, for the nozzle were discussed. Results show that the flow dynamics are most sensitive to changes of $R_{c h}$ among of $R_{c h}, R_{c w}$, and $R_{d w}$, The entrainment ratio $\left(R_{e n}\right)$ increases with $R_{c h}, R_{d w}$, and $R_{d w}$. The primary and secondary masses decrease with increasing $R_{c h}$ and $R_{d w}$. The primary and secondary masses increase with $R_{d w}$. The conclusions obtained in the study provide guidelines for designing a rectangular slot nozzle for pulse jet cleaning of the filter elements.

(Received in June 2015, accepted in December 2015. This paper was with the authors 3 months for 1 revision.)
\end{abstract}

Key Words: Numerical Simulation, Three-Dimensional Model, Rectangular Nozzle, Pulse Cleaning, Entrainment Effect

\section{INTRODUCTION}

Removal of dust cake from the surface of filter elements is a major task in cleaning filter elements, which are used in numerous applications [1, 2]. One of the most effective and common technologies used to clean filter elements is the reverse pulse jet cleaning method [37]. The momentum of the pulse gas in the filter cavity should be sufficiently and uniformly formed along the length of the filter element to prevent patch cleaning [8]. A dead zone usually forms near the mouth of the filter element where the effect of the pulse cleaning is more negligible than in the surrounding portion because of the formation of negative pressure [9]. The jet nozzle blows a high-speed primary jet into the filter vessel to form the primary flow of pulse gas. The primary pulse gas creates a low-pressure zone around the pulse nozzle and generates suction momentum of the surrounding fluid, which induces a secondary airflow $[10,11]$. The formation of the secondary gas flow is advantageous in a few ways to the operation of the filter unit. First, the cleaning effect of the filter element is increased with the additional gas injection into the filter cavity, and the reverse momentum for detaching the dust cake is increased on the surface of the filter element. Second, the entrained hot gas by the secondary gas flow increases the temperature of the pulse gas that enters the filter cavity and reduces the thermal shock during the injection of the cold pulse gas [8]. However, a drawback is that negative pressure forms and usually occurs near the mouth of the filter element when the suction effect is prolonged farther into the filter element. Thus, the formation of sufficient and uniform pulse pressure is important to ensure the best cleaning conditions of the filter element during the pulse jet cleaning process. 
The best way to effectively utilize the pulse gas is to maximize the entrainment effect at the guiding area (between the nozzle exit and the diffuser mouth) of the pulse gas, where the negative pressure is formed to prevent the negative effect due to the extension of the negative tendency to the diffuser. Generally, nozzle size shows the reciprocal effect, which increases the primary pulse gas mass while decreasing the secondary pulse mass as the size increases [8]. The convergent nozzle showed a higher pulse effect than the straight nozzle because of its concentrating effect, which causes higher gas speeds.

\section{STATE OF THE ART}

To clarify the nature of gas flow during pulse cleaning, some researchers have used the computational fluid dynamics (CFD) software FLUENT on a two-dimensional microscopic basis [12, 13]. Zhang et al. [14] showed the pressure oscillation in the nozzle outlet and the pressure oscillation spread in the pulse cleaning flow field with the CFD calculations. To overcome the experimental limitations in obtaining the design values of the pulse cleaning system, several scholars performed CFD analysis. These studies focused on the optimization of the pressure of pulse gas tank [7-9], mass flow rate [9], pulse duration [9, 12], and the distance from nozzle to filter [2,9], among others. Mohammed [15] compared the numerical simulation obtained from three different turbulence models with the experimental data to validate the FLUENT code. The validation showed that the standard $k-\varepsilon$ turbulence model can successfully simulate the nozzle and diffuser.

However, most of the abovementioned studies were limited to tubular nozzles for the single filter element and seldom focused on the group filter elements and the rectangular slot nozzle. Small tubular nozzles located on a tubular manifold or a large tubular nozzle for a venturi-type diffuser [16] have been recently used for the group filter element unit in industrial fields. The former is relatively effective but requires a complicated arrangement of the pulse cleaning system. The latter was also effective but consumed considerable space in the filter unit because of its shape. A cake pie-type diffuser provides an attractive structure that covers the hundred filter element on a single diffuser. The pulse gas of high pressure is distributed above the filter elements during its propagation in the radial direction of the diffuser. The pulse cleaning system with a rectangular slot nozzle is compact and requires the least space.

One of the important tasks of this system is how to achieve the maximum pulse gas leading into the rectangular receiver of the cake pie-type diffuser. The expected results from the experimental study are restrictive because of the deficits of the exact measurement skills and several co-dependent factors that influence the pulse effect. Thus, CFD is used in this study to evaluate the effect of the shape and dimension of a rectangular slot nozzle on the performance of the pulse gas.

\section{METHODOLOGY}

\subsection{Nozzle}

The novel nozzle has a venturi shape. Fig. 1 shows the primary standard novel nozzle used in the experiment. The schematic nozzle is shown in Fig. 2. This nozzle receives the pulse gas at the rectangular mouth, which has a width of $80 \mathrm{~mm}$, a height of $30 \mathrm{~mm}$, and a length of 200 $\mathrm{mm}$, where the nozzle starts to converge along $60 \mathrm{~mm}$ up to the throat, which has a width of $60 \mathrm{~mm}$. The nozzle diverges along $90 \mathrm{~mm}$ and then converges along $60 \mathrm{~mm}$. Thus, the nozzle height is constantly contracted to the end point from $30 \mathrm{~mm}$ along the length of $210 \mathrm{~mm}$. The convergent ratios in the narrow direction $\left(R_{c h}\right)$ and the wide direction $\left(R_{c w}\right)$, as well as the divergent ratio at the diverging section $\left(R_{d w}\right)$ of the nozzle, were defined as Eqs. (1), (2), and 
(3) respectively. The variables and parameters in this model are shown in Fig. 2. The primary standard novel nozzle has the reference dimensions of 30,60 , and $90 \mathrm{~mm}$ for directions of the narrow height of the nozzle mouth $(h)$, width $(w)$, and the expended position for nozzle contraction (e), respectively.

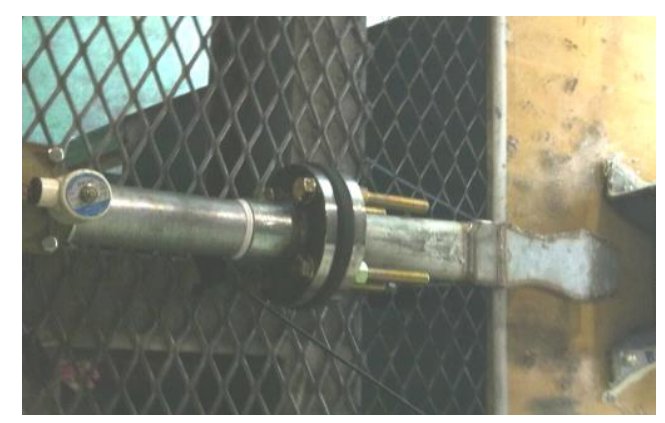

Figure 1: Experiment setup for pulse cleaning system.

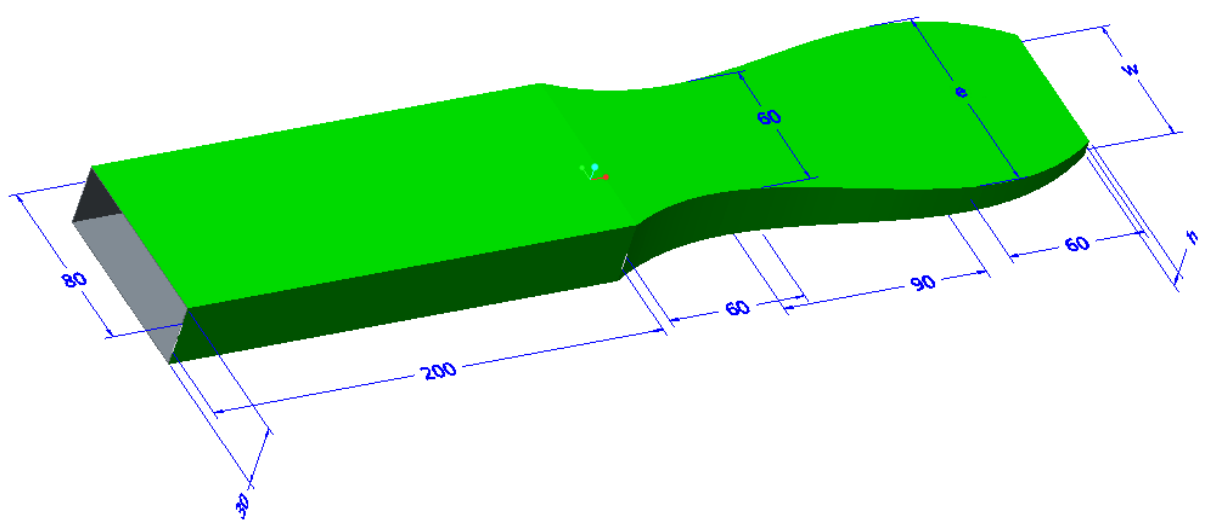

Figure 2: Schematic of nozzle size and dimension varied in simulation model.

$$
\begin{aligned}
& R_{c h}=\frac{30-h}{30} \\
& R_{c w}=\frac{90-w}{90} \\
& R_{d w}=\frac{e-60}{60}
\end{aligned}
$$

\subsection{Geometry modelling and grid pre-processing}

A three-dimensional model was applied in this work. The flow direction of pulse gas along the centreline is determined as the $x$-axis for the rectangular coordinates. The narrow and wide directions from the centre point are $y$ - and $z$-axes, respectively. Given the symmetry of the flow problem, only a quarter of the domain needs to be modelled. The pre-processing, including the geometry model and structural mesh generation, was completed in GAMBIT. To accurately simulate the flow dynamic performance of the nozzle, further mesh refinement of the calculation domain is required. The meshing sizes in the calculation domain are strictly controlled, and particular refinements have been made. In all CFD simulations, a mesh dependence test is crucial to check the convergence of the computational results with respect to spatial resolution [17]. The mesh dependence test is performed by refining the mesh to its final configuration. The grid system consisted of structured quadrilateral cells. Fig. 3 demonstrates the results of the grid independence study based on the static pressure along the nozzle axis. Four sets of grids were used to find sufficient grid points. The first (coarse) grid 
has 367,000 cells. The second (medium) grid set has 386,000 cells. The third (fine) set grid has 386,000 cells, which are generated by the same minimum space as the second set but the grid increasing ratio changed, thereby making the grid near the nozzle outlet denser than the second grid. The fourth grid with 706,000 cells is denser than the third grid but shows no obvious differences in pressure distribution. Therefore, the third grid set is determined as the independent grid that is considered sufficient for the remaining cases and is used in this work. The independent grid is shown in Fig. 4.

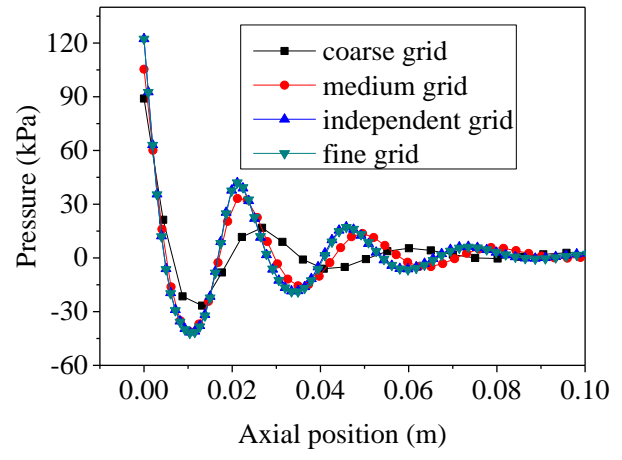

Figure 3: Grid independent examination.

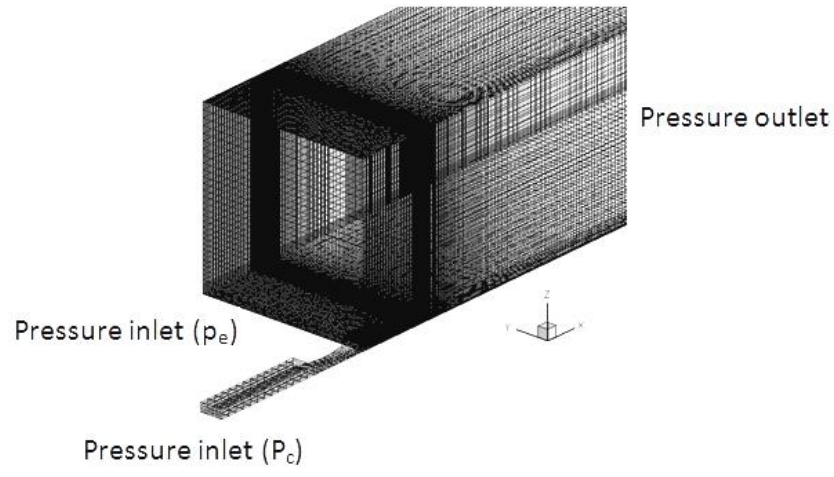

Figure 4: Meshing of the computational domain.

\subsection{Governing equations}

CFD is fundamentally based on the governing equations of fluid dynamics. These captions represent mathematical statements of the conservation laws of physics, where the following physical laws are adopted:

(1) Mass is conserved for the compressible fluid and can be written as:

$$
\frac{\partial \rho}{\partial t}+\frac{\partial}{\partial x_{i}}\left(\rho u_{i}\right)=0
$$

(2) For the fluid analysis of the entire calculation domain, the expression of balance of momentum is given in the following form:

$$
\frac{\partial}{\partial t}\left(\rho u_{i}\right)+\frac{\partial}{\partial x_{j}}\left(\rho u_{i} u_{j}\right)=-\frac{\partial p}{\partial x_{i}}+\frac{\partial}{\partial x_{j}}\left[\mu_{e f f}\left(\frac{\partial u_{i}}{\partial x_{j}}+\frac{\partial u_{j}}{\partial x_{i}}-\frac{2}{3} \delta_{i j} \frac{\partial u_{l}}{\partial x_{l}}\right)\right]+\frac{\partial}{\partial x_{j}}\left(-\rho \overline{u_{i} u_{j}^{\prime}}\right)
$$

(3) The modelled energy equation is:

$$
\frac{\partial}{\partial t}(\rho E)+\frac{\partial}{\partial x_{i}}\left(u_{i}(\rho E+p)\right)=\frac{\partial}{\partial x_{i}}\left[\left(\alpha+\frac{c_{p} \mu_{t}}{p_{r t}}\right) \frac{\partial T}{\partial x_{i}}+u_{j}\left(\tau_{i j}\right)_{e f f}\right]
$$

where $E$ and $T$ are energy and temperature. $\tau_{i j}$ is the stress tensor, defined as:

$$
\tau_{i j}=\left[\mu\left(\frac{\partial u_{i}}{\partial x_{j}}+\frac{\partial u_{j}}{\partial x_{i}}\right)\right]-\frac{2}{3} \mu \frac{\partial u_{l}}{\partial x_{l}} \delta_{i j}
$$

The term $\delta_{i j}$ is the viscous heating due to the dissipation. The equation of state for perfect gases is added to close the system:

$$
p=\rho R T
$$

Turbulence models used in this paper rely on the Boussinesq hypothesis, which relates the Reynolds stress and the averaged velocity gradient. That is, both are based on an eddy viscosity assumption, which makes the Reynolds stress tensor from equation averaging proportional to the mean deformation rate tensor:

$$
-\rho \overline{u_{i}^{\prime} u_{j}^{\prime}}=\mu\left(\frac{\partial u_{i}}{\partial x_{j}}+\frac{\partial u_{j}}{\partial x_{i}}\right)-\frac{2}{3}\left(\rho k+\mu_{t} \frac{\partial u_{i}}{\partial x_{i}}\right) \delta_{i j}
$$


The simplest complete models of turbulence are two-equation models of the standard $k-\varepsilon$ model, where the solution of two separate transport equations allows the turbulent velocity and length scales to be independently determined. The standard $k-\varepsilon$ model has become the workhorse of practical engineering flow calculations since it was proposed by Launder and Spalding. The robustness, economy, and reasonable accuracy of this model for a wide range of turbulent flows explain its popularity in industrial flow and heat transfer simulations [18].

The turbulence kinetic energy $(k)$ and its rate of dissipation $(\varepsilon)$ are obtained from the following transport equations:

and

$$
\frac{\partial(\rho k)}{\partial t}+\frac{\partial}{\partial x_{i}}\left(\rho k u_{i}\right)=\frac{\partial}{\partial x_{j}}\left[\left(\mu+\frac{\mu_{t}}{\sigma_{k}}\right) \frac{\partial k}{\partial x_{j}}\right]+G_{k}+G_{b}-\rho \varepsilon-Y_{M}
$$

$$
\frac{\partial(\rho \varepsilon)}{\partial t}+\frac{\partial}{\partial x_{i}}\left(\rho \varepsilon u_{i}\right)=\frac{\partial}{\partial x_{j}}\left[\left(\mu+\frac{\mu_{t}}{\sigma_{\varepsilon}}\right) \frac{\partial \varepsilon}{\partial x_{j}}\right]+C_{1 \varepsilon} \frac{\varepsilon}{k}\left(G_{k}+C_{3 \varepsilon} G_{b}\right)-C_{2 \varepsilon} \rho \frac{\varepsilon^{2}}{k}
$$

In these equations, $G_{k}$ represents the generation of turbulence kinetic energy attributed to the mean velocity gradients. $G_{b}$ is the generation of turbulence kinetic energy attributed to buoyancy. $Y_{M}$ represents the contribution of the fluctuating dilatation in compressible turbulence to the overall dissipation rate. $C_{\varepsilon 1}, C_{\varepsilon 2}$, and $C_{\varepsilon 3}$ are constants. $\sigma_{k}$ and $\sigma_{\varepsilon}$ are the turbulent Prandtl numbers for $k$ and $\varepsilon$, respectively.

\subsection{Numerical scheme and solution convergence}

The Reynolds-averaged compressible Navier-Stokes equations are solved with a $k-\varepsilon$ turbulence model for closure by a controlled volume approach with the commercial CFD package FLUENT 6.3. The numerical boundary conditions of the inlet pressure and outlet pressure outlet are applied. A quarter of the nozzle is modelled in consideration of the symmetrical structure of the simulation domain to save calculation time. Therefore, the symmetry boundary condition is applied in this work. Dry air with ideal gas approximation is used as working fluid. All the walls are considered to be adiabatic with no slip. For all equations, convective terms are discretized by a second-order upwind scheme.

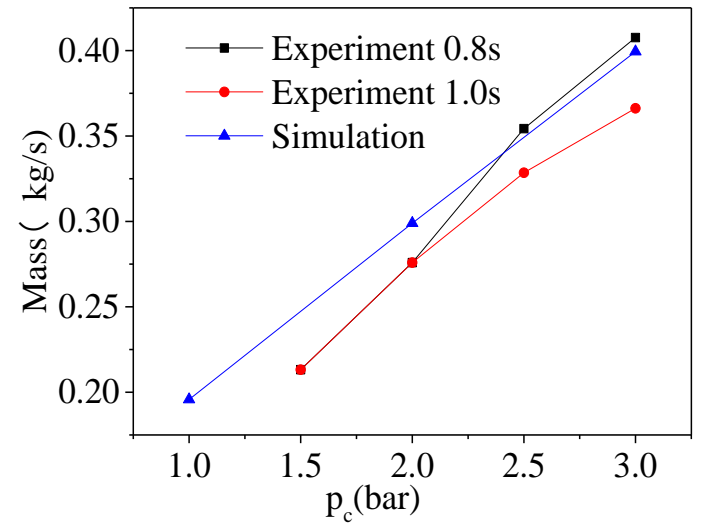

Figure 5: Model validation.

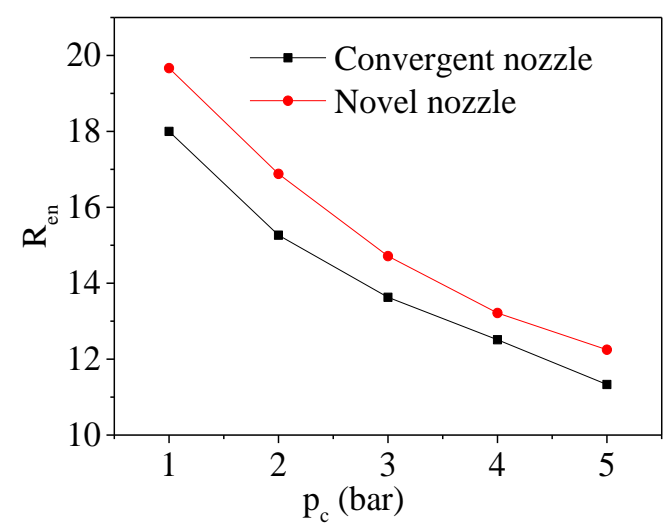

Figure 6: Comparison of convergent nozzle and current nozzle.

The mass flow rates through the nozzle outlet obtained from the numerical simulation agree with the experimental values for the reference nozzle, as shown in Fig. 5. The experiment was conducted on two cases where the pulse duration was 0.8 and $1.0 \mathrm{~s}$, respectively. The mass flow rate in the experiment is calculated by measuring the pressure difference of the air tank during the pulse duration. The mean mass flow will be more 
accurate with the increase of the pulse duration time as the operational end effect of the pulse valve should be reduced because of the longer opening time. However, maintaining the constant pressure of the pulse tank when the pulse duration time increases is difficult as the air supply into the pulse tank is initially interrupted to measure the air consumption. Thus, the mass flow rate in the experimental calculation decreased when the pulse duration increased. The calculated mass flow rate is evidently in good agreement with the experimental mass flow rate by considering the experimental uncertainty. Therefore, the proposed simulation model is useful to predict the flow dynamics of the pulse gas from the nozzle tip.

\section{SIMULATION ANALYSIS AND DISCUSSION}

\subsection{Primary novel nozzle}

The performance of a primary novel nozzle of the venturi-type is compared with that of a conventional convergent nozzle with a gradual contraction type with the same dimensions. The primary mass that approaches the nozzle mouth is the same for each nozzle. The performance was compared with the entrainment effect. The entrainment ratio is an effective parameter to evaluate the pulse cleaning effect of the nozzle system as defined in Eq. (12), where $R_{e n}$ is the entrainment ratio; $m_{1}$ is the gas mass from nozzle outlet (primary mass), which is obtained by integrating the velocity and density of pulse gas in the area of nozzle outlet; $m_{2}$ is the mass of the entrained gas (secondary mass); and $m_{t}$ is the total mass, which is equal to the summation of $m_{1}$ and $m_{2} . m_{t}$ is the integration of gas velocity and density in the area of the outlet of the computational domain. Fig. 6 shows that the entrainment ratio of the current novel nozzle is slightly higher than that of the convergent nozzle.

$$
\mathrm{R}_{\mathrm{e} n}=\frac{m_{2}}{m_{1}}=\frac{m_{t}-m_{1}}{m_{1}}
$$

To learn the dynamic behaviour of pulse gas leaving the outlet of the primary standard novel nozzle, detailed results for the primary rectangular nozzle are provided in Fig. 7: $R_{c h}=0.77, R_{c w}=0.33$, and $R_{d e}=0.5$. Fig. 7 shows the velocity vector distribution near the nozzle outlet at plane $z=0$, which illustrates the entrainment effect. The surrounding gas flows toward the primary pulse gas and then combines with the primary gas as the total pulse cleaning gas. The entrained secondary gas flow is strongly advantageous to the operation of the filter unit.

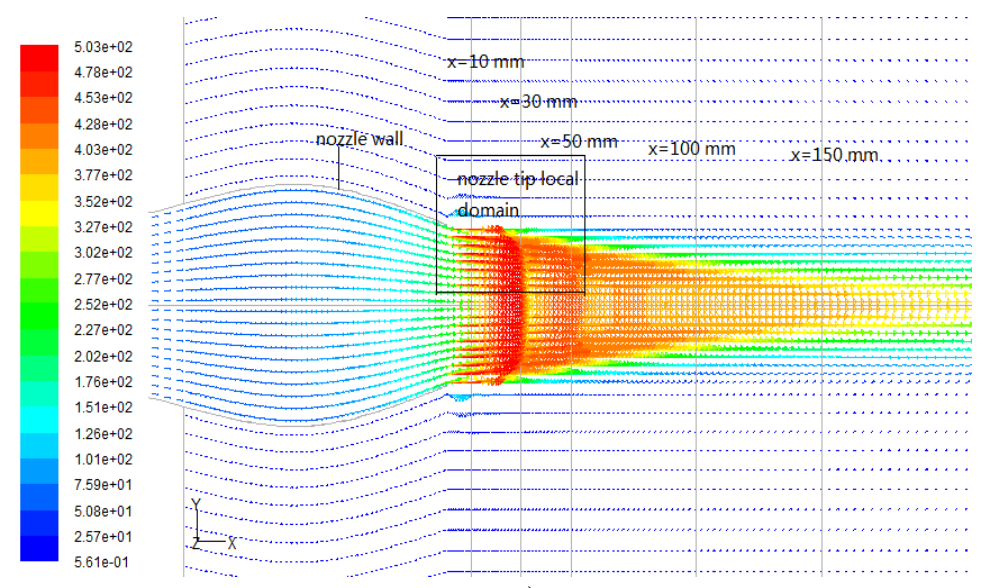

a)

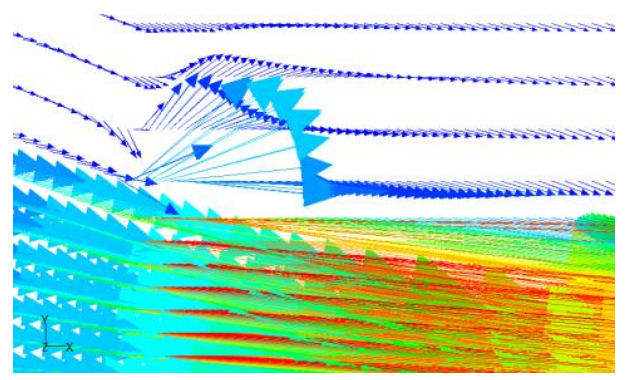

b)

Figure 7: Velocity vector of the pulse gas stream around the nozzle outlet, a) velocity vectors distribution, b) magnification of velocity vectors around the very close domain of nozzle. 


\subsection{Effect of nozzle convergent ratio in the narrow direction $\left(R_{c h}\right)$}

The effect of nozzle convergence was inspected by changing the height of the nozzle tip while maintaining constant dimensions of the rectangular nozzle mouth (height, $30 \mathrm{~mm}$; width, $80 \mathrm{~mm})$. The nozzle gradually converges to a determined height $(1-11 \mathrm{~mm})$ from its $30 \mathrm{~mm}$ high mouth along a distance of $210 \mathrm{~mm}$, as shown at Fig. 1. The convergent ratio in the narrow direction is defined as Eq. (1) for this case. Figs. 8 and 9 show the contour plots of pressure and the Mach number, respectively. Shock structures in the supersonic nozzle geometries are investigated for a pulse cleaning pressure of $p_{c}=5$ bar. No shock waves occur in the case of $R_{c h}=0.97$. The length of the shock wave increases as $R_{c h}$ decreases from 0.97 to 0.5 .

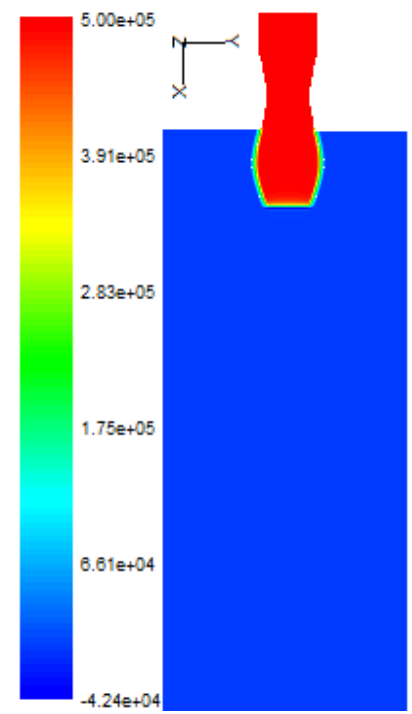

a)

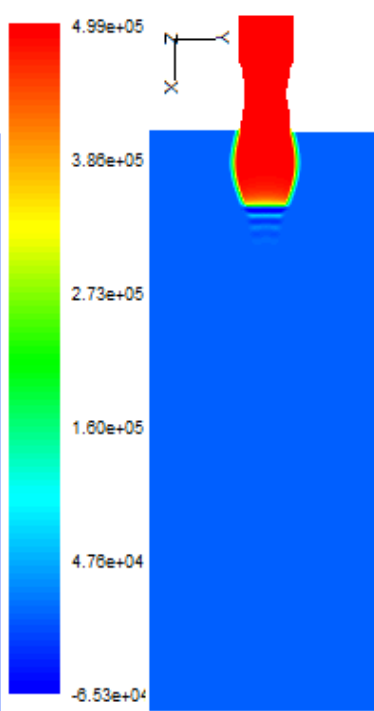

b)

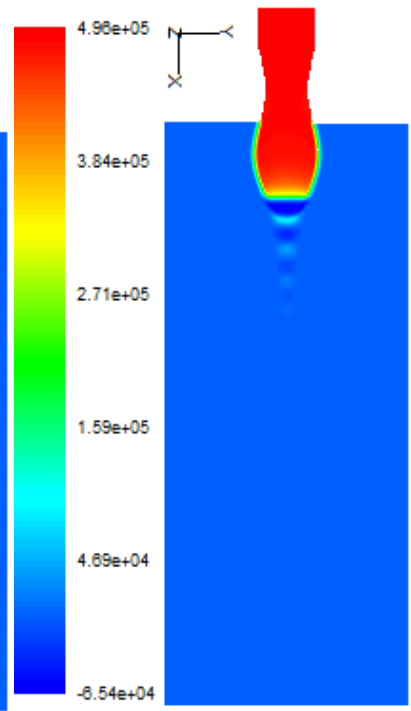

c)

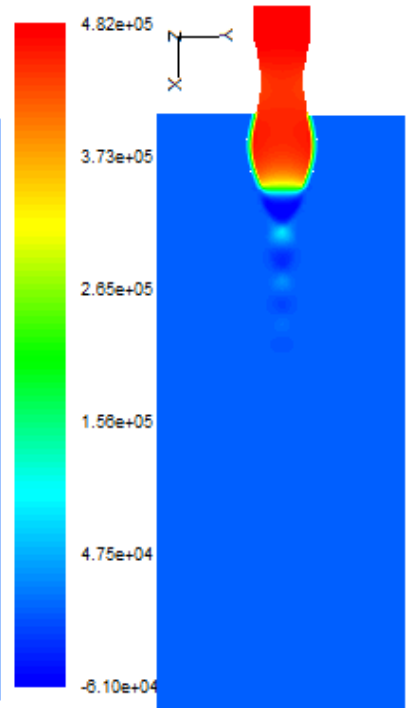

d)

Figure 8: Contour plots of pressure in plane $z=0$ at different convergent ratio in narrow direction at $p_{c}=5$ bar; a) $R_{c h}=0.97$, b) $R_{c h}=0.9$, c) $R_{c h}=0.77$, d) $R_{c h}=0.5$.

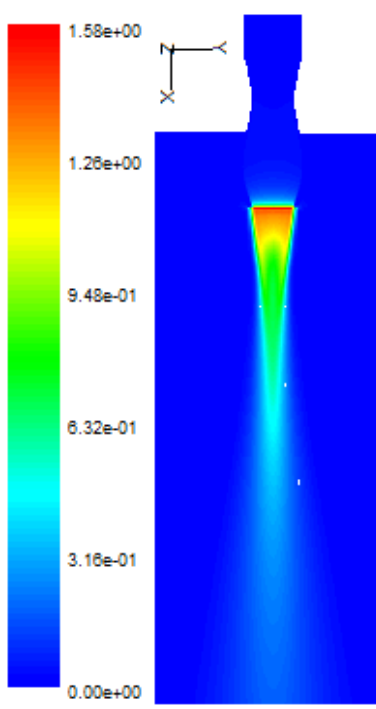

a)

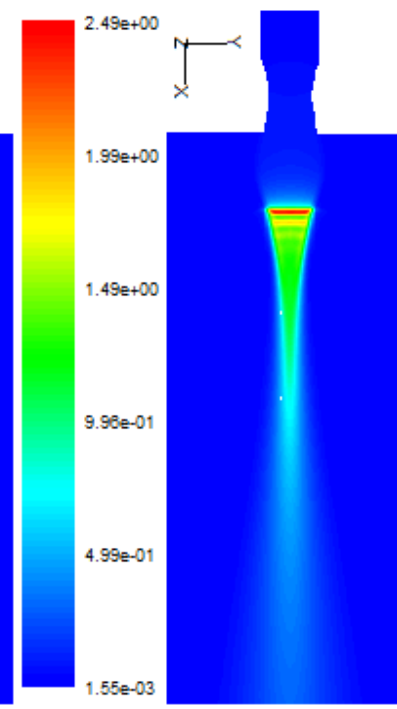

b)

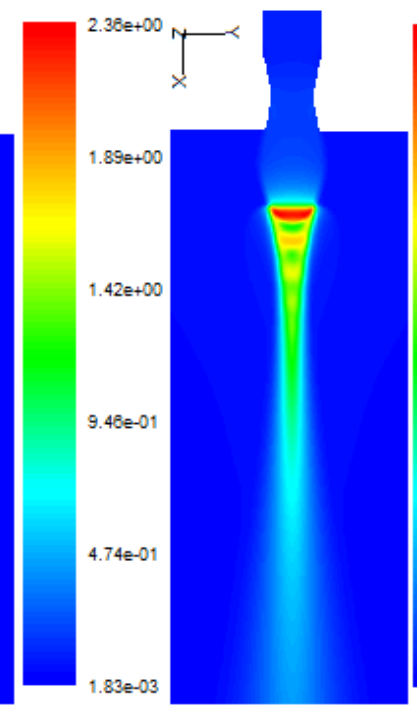

c)

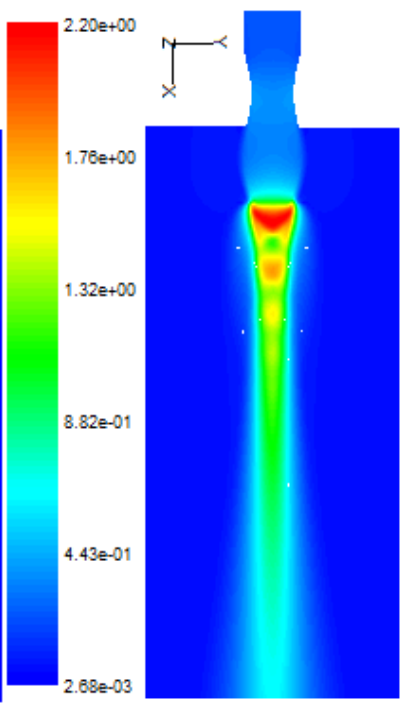

d)

Figure 9: Contour plots of Mach number in plane $z=0$ at different convergent ratio in narrow direction at $p_{c}=5$ bar; a) $R_{c h}=0.97$, b) $R_{c h}=0.9$, c) $R_{c h}=0.77$, d) $R_{c h}=0.5$.

The variations of the entrainment ratio $\left(R_{e n}\right)$ with the convergent ratio of the nozzle in the narrow direction $\left(R_{c h}\right)$ are shown in Fig. 10 as the pulse cleaning pressure $\left(p_{c}\right)$ changed from 1 
bar to 5 bar. Fig. 11 shows that the entrainment effect increased as the convergent ratio of the nozzle in the narrow direction $\left(R_{c h}\right)$ increased, but this effect decreased as the pulse pressure increased. The increase of $R_{c h}$ decreased the nozzle outlet area, which induced a faster velocity of the pulse gas leaving the nozzle tip. The kinematic energy of the fluid increased, which induced the reduction of potential energy to form negative pressure in the area in close proximity to the nozzle tip compared with the surrounding pressure. As shown in Fig. 8, the formation of the negative pressure in the fluid domain was the main source of the entrainment effect.

The effect of $R_{c h}$ on the entrainment ratio was dominant when $R_{c h}$ was larger than 0.8 , as shown in Fig. 10, and corresponds to a height of less than $6 \mathrm{~mm}$. However, the absolute value of the entrainment gas actually decreased as $R_{c h}$ increased, as shown in Fig. 11. In this figure, the entrained mass parabolically decreased while the primary mass linearly decreased as the $R_{c h}$ increased. The increasing rate of the entrainment ratio was almost exponential with $R_{c h}$. The large value of the entrainment effect in the lower nozzle height stemmed from the lower $m_{1}$, which had a large calculation value because of the relatively and arithmetically smaller denominator in this region. These phenomena also occurred in the case of the pulse pressure effect, as shown in Fig. 11, where the higher entrainment ratio appeared at a lower pulse pressure. However, a larger pulse mass formed when the nozzle was wider and the pulse pressure was higher. Therefore, finding a certain criterion for the selection of the optimum nozzle height to provide the maximum amount of pulse gas is difficult. A greater nozzle height and a higher pulse pressure correspond to a greater amount of pulse gas.

Higher entrainment is desirable to prevent the thermal shock of filter elements that work at high temperature because of its mixing effect. However, given the heating effect of the primary cold gas with the surrounding entrained hot gas, reducing the thermal shock of the filter element working at high temperature is important. Thus, the entrainment effect remains useful in evaluating the effectiveness of the pulse cleaning system.

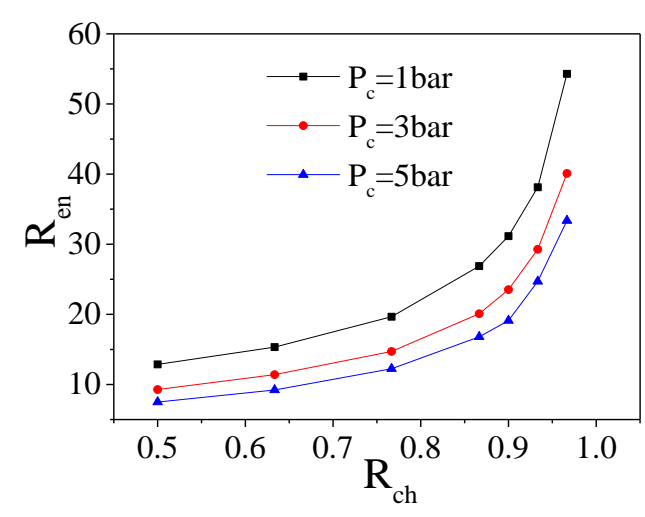

Figure 10: Variations of $R_{e n}$ with $R_{c h}$.

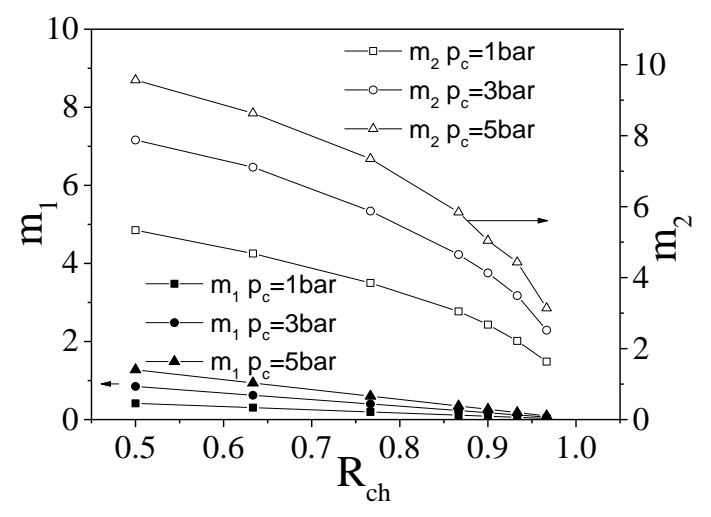

Figure 11: Variations of $m_{1}$ and $m_{2}$ with $R_{c h}$.

\subsection{Effect of nozzle convergent ratio in wide direction $\left(\boldsymbol{R}_{c w}\right)$}

The effect of the convergent ratio in the wide direction $\left(R_{c w}\right)$ was inspected by changing the width of the nozzle tip by keeping the height of the nozzle tip at $7 \mathrm{~mm}$. The nozzle gradually converged into a determined width (30-90 mm corresponding to $R_{c w}$ of 0.67 to 0 , respectively) from its extension width of $90 \mathrm{~mm}$ over a distance of $60 \mathrm{~mm}$, as shown in Fig. 1. The convergence ratio was defined as Eq. (2) for this case.

Contour plots of the pressure and Mach number in plane $z=0 \mathrm{~mm}$ at different $R_{c w}$ are shown in Figs. 12 and 13, respectively. The width of the shock wave increased as the convergent ratio in the wide direction $\left(R_{c w}\right)$ decreased. The length of the shock wave is nearly constant when the convergent ratio in the wide direction $\left(R_{c w}\right)$ decreased from 0.67 to 0 . The effect region increased with the decreasing $R_{c w}$. 


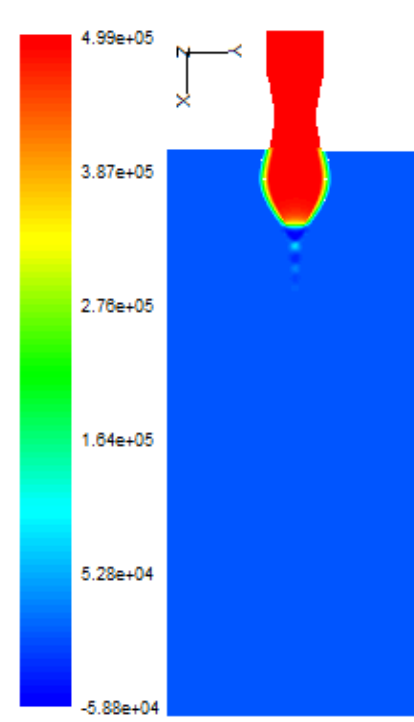

a)

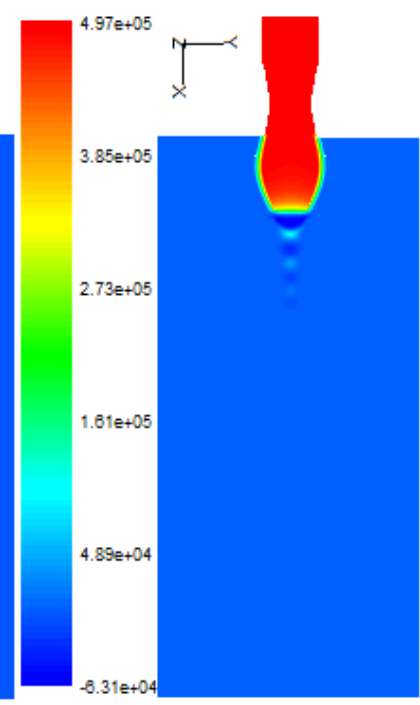

b)

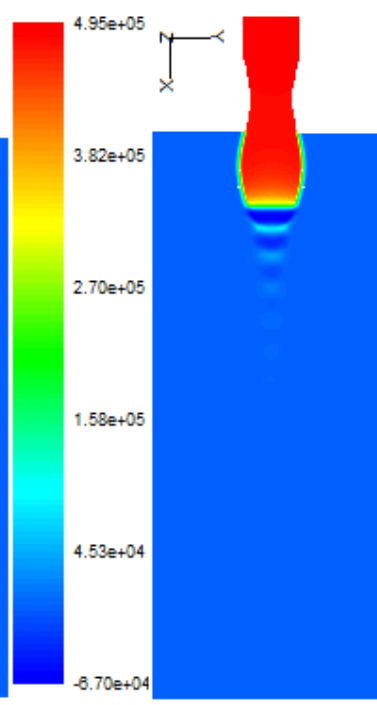

c)

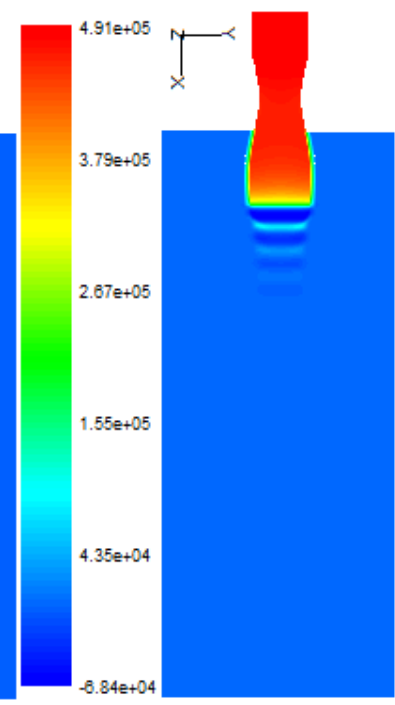

d)

Figure 12: Contour plots of pressure in plane $z=0$ at different convergent ratio in wide direction at $p_{c}=5$ bar; a) $R_{c w}=0.67$, b) $R_{c w}=0.44$, c) $R_{c w}=0.22$, d) $R_{c w}=0$.

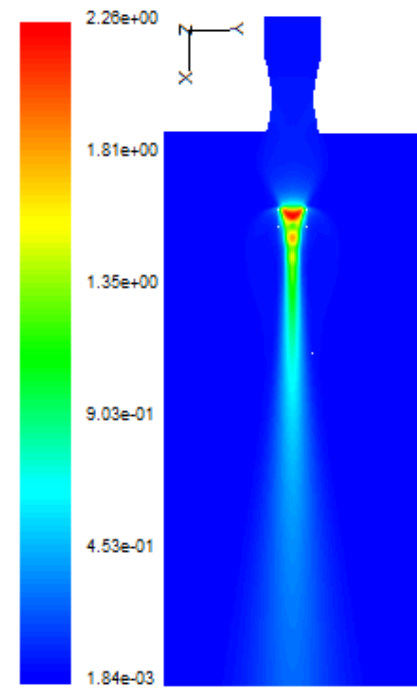

a)

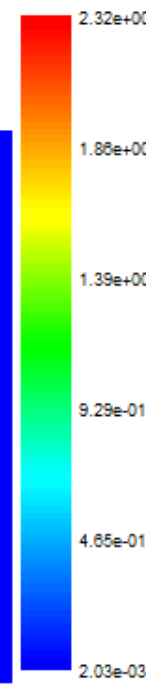

$2.03 \mathrm{e}-03$

b)

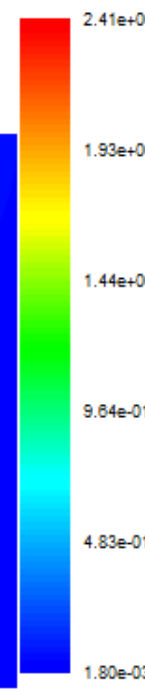

)

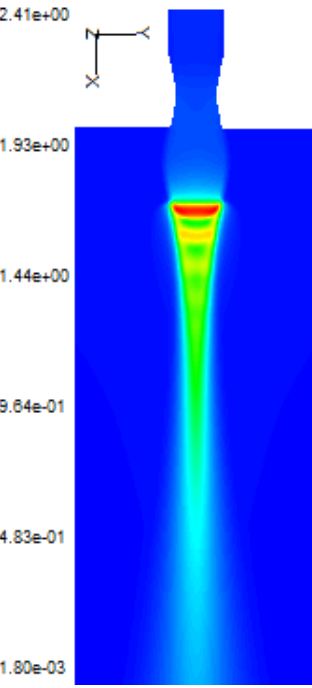

c)

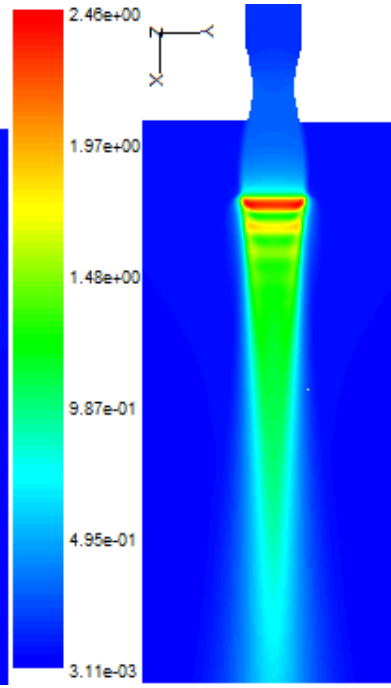

d)

Figure 13: Contour plots of Mach number in plane $z=0$ at different convergent ratio in wide direction at $p_{c}=5 \mathrm{bar} ;$ a) $\left.R_{c w}=0.67, \mathrm{~b}\right) R_{c w}=0.44$, c) $R_{c w}=0.22$, d) $R_{c w}=0$.

Fig. 14 shows the effect of the convergent ratio in the wide direction $\left(R_{c w}\right)$ on the entrainment ratio at different pulse cleaning pressures. The entrainment ratio gradually increased with the increasing $R_{c w}$ from 0 to 0.67 . The entrainment ratio decreased with the pulse cleaning pressure $p_{c}$. Fig. 15 shows the variation of the primary mass $\left(m_{1}\right)$ and secondary mass $\left(m_{2}\right)$ with the convergent ratio in the wide direction $\left(R_{c w}\right)$ when the pulsed cleaning gas pressure is 3 bar. The primary mass and secondary mass decrease with the increase of $R_{c w}$. Increasing the nozzle outlet width can improve the primary gas and entrained gas because the entrainment ratio gradually decreased when the nozzle outlet width increased.

The variation trends of the shock region, $R_{e n}, m_{1}$, and $m_{2}$ show opposite trends with $R_{c w}$. A suitable convergent ratio in the wide direction is necessary because the shock region, $R_{e n}, m_{1}$, and $m_{2}$ are all important factors in the pulse cleaning system. 


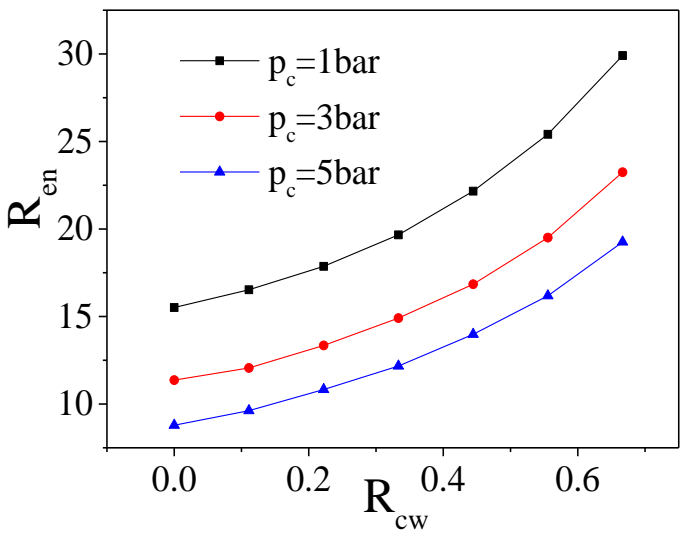

Figure:14: Variations of $\mathrm{R}_{\mathrm{en}}$ with $\mathrm{R}_{\mathrm{cw}}$.

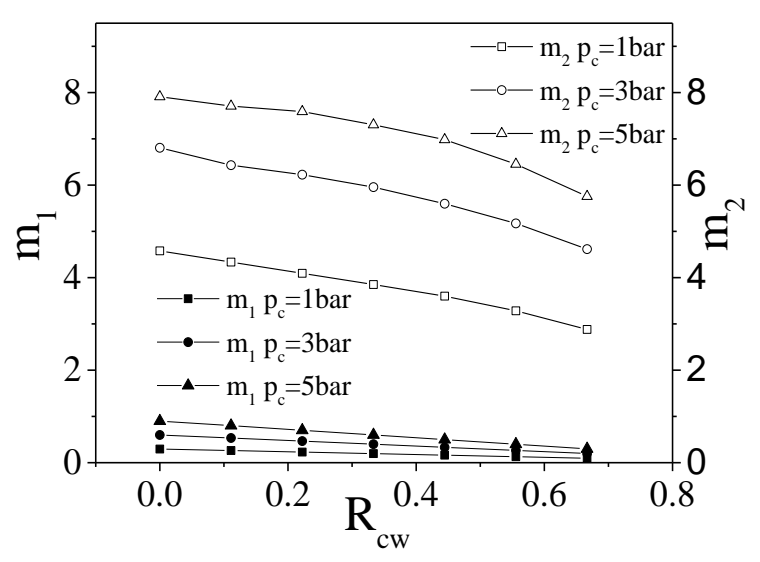

Figure15: Variations of $\mathrm{m}_{1}$ and $\mathrm{m}_{2}$ with $\mathrm{R}_{\mathrm{cw}}$.

\subsection{Effect of nozzle divergent ratio in wide direction $\left(\boldsymbol{R}_{d w}\right)$}

The effect of nozzle divergence was inspected by changing the nozzle extension width, keeping the rectangular nozzle mouth dimensions constant at a height of $30 \mathrm{~mm}$ and width of $80 \mathrm{~mm}$, and keeping the rectangular nozzle tip dimensions constant at a height of $7 \mathrm{~mm}$ and width of $60 \mathrm{~mm}$. The nozzle gradually diverged to a determined width $(60-110 \mathrm{~mm})$ from its throat width of $60 \mathrm{~mm}$ along the distance of $90 \mathrm{~mm}$, as shown in Fig. 1. The divergence ratio is defined by Eq. (3) for this case.

Figs. 16 and 17 show the shock wave structures in terms of the contour plots of pressure and Mach number at different divergent ratios at the diverging section $\left(R_{d w}\right)$ of the nozzle. The divergent ratio $\left(R_{d w}\right)$ barely affects the number of shock waves downstream of the nozzle tip, although the width of the shock wave decreases as the divergent ratio $\left(R_{d w}\right)$ increases from 0 to 0.83 . The divergent ratio at the diverging section has no obvious effect on the shock shape and strength.

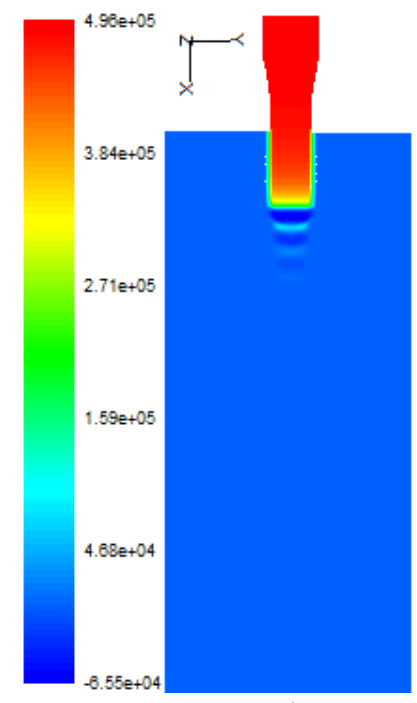

a)

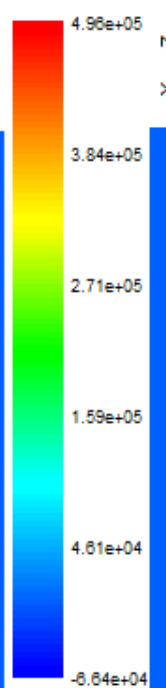

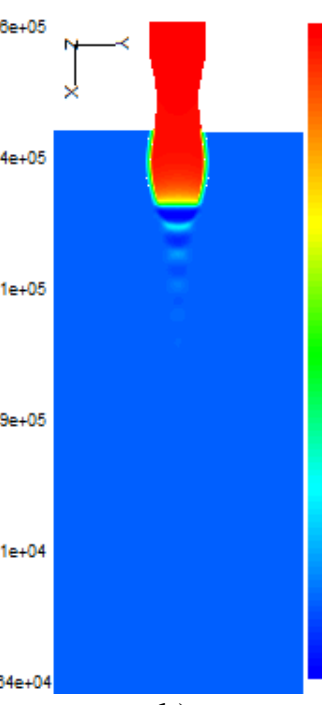

b)

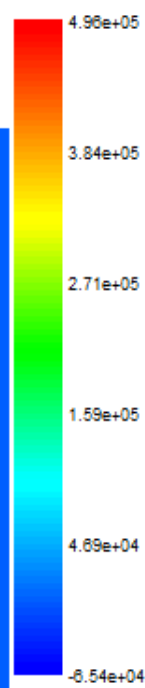

$8.54 \mathrm{e}+0$

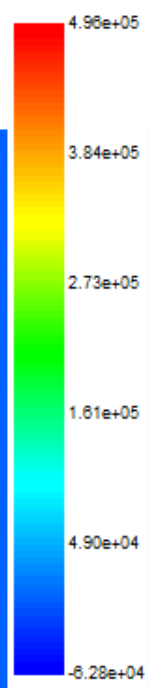

)

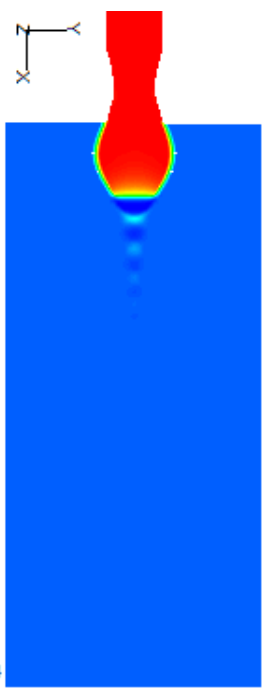

d)

Figure 16: Contour plots of pressure in plane $z=0$ at different divergent ratio at the diverging section at $p_{c}=5$ bar; a) $R_{d e}=0$, b) $R_{d e}=0.33$, c) $R_{d e}=0.5$, d) $R_{d e}=0.83$. 


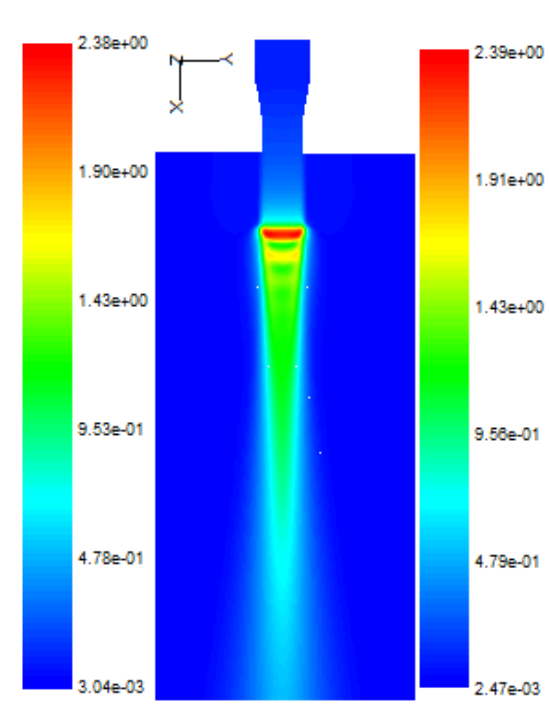

a)

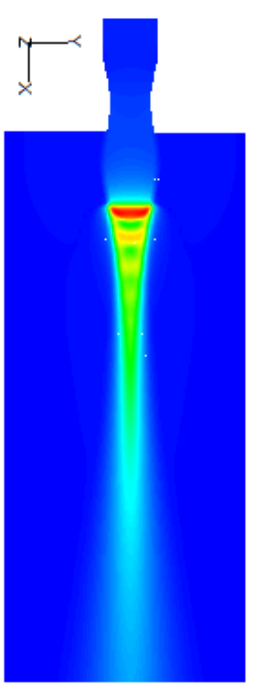

b)

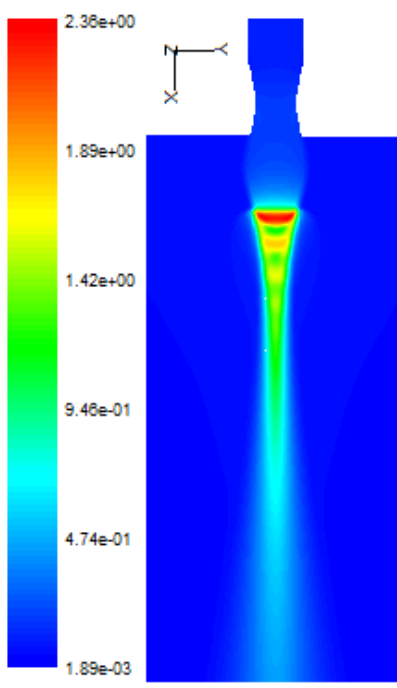

c)

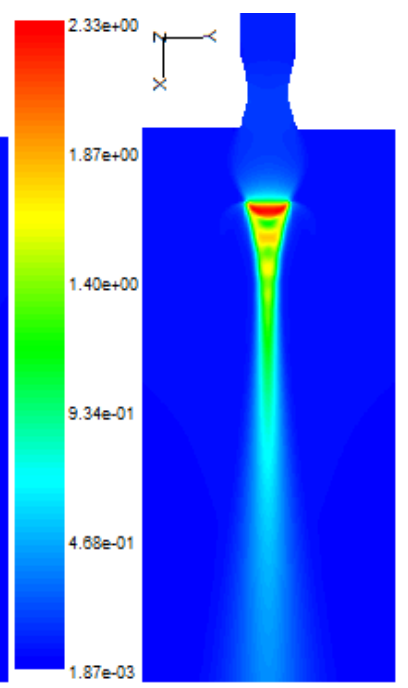

d)

Figure 17: Contour plots of Mach number in plane $z=0$ at different divergent ratio at the diverging section at $p_{c}=5$ bar; a) $R_{d e}=0$, b) $R_{d e}=0.33$, c) $R_{d e}=0.5$, d) $R_{d e}=0.83$.

Fig. 18 shows the effect of the divergent ratio at the diverging section $\left(R_{d w}\right)$ of the nozzle on the entrainment ratio at different pulse cleaning pressures. The entrainment ratio increased with the increasing nozzle divergent ratio at the diverging section and with the decreasing pulse cleaning pressure. The entrainment ratio first increases slightly when $R_{d w}$ is less than 0.33 before the entrainment ratio gradually increases when $R_{d w}$ exceeds 0.5 . The lower pulse cleaning pressure causes the higher entrainment ratio, although the higher pulse cleaning pressure will entrain more surrounding gas than the lower pulse cleaning pressure. Fig. 19 shows the variation of the primary mass $\left(m_{1}\right)$ and secondary mass $\left(m_{2}\right)$ with the divergent ratio at the diverging section $\left(R_{d w}\right)$ when the pulse cleaning gas pressure is 3 bar. The primary mass $\left(m_{1}\right)$ remains constant when $R_{d w}$ increases from $60 \mathrm{~mm}$ to $110 \mathrm{~mm}$. The secondary mass $\left(m_{2}\right)$ gradually increases with the increase of $R_{d w} . R_{d e}$ is the least sensitive parameter among $R_{c h}$, $R_{c w}$, and $R_{d e}$.

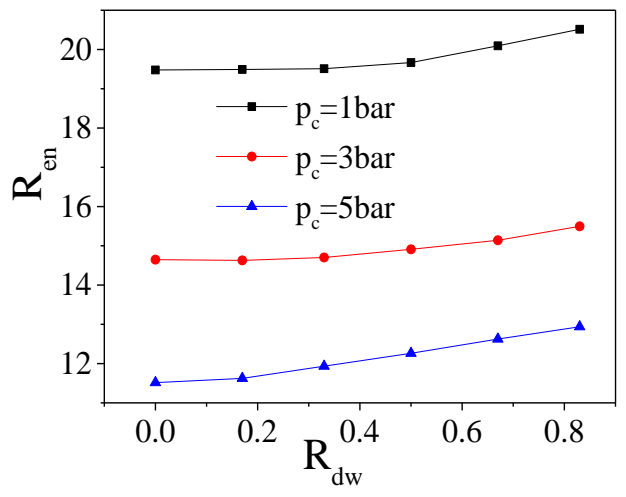

Figure 18: Variations of $R_{e n}$ with $R_{d w}$.

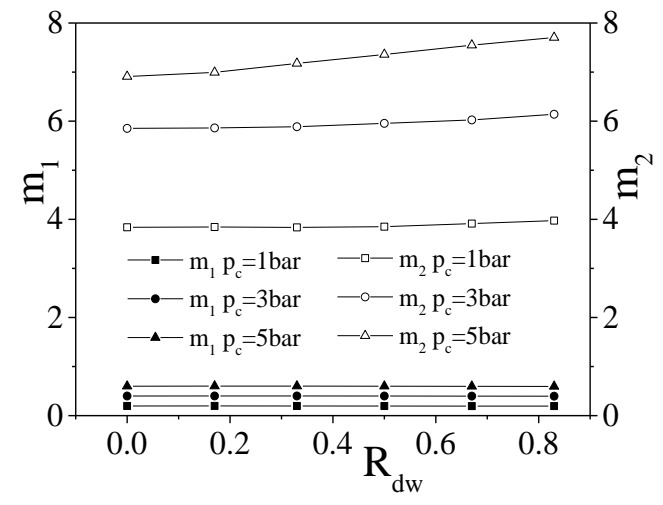

Figure 19: Variations of $m_{1}$ and $m_{2}$ with $R_{w d}$.

\section{CONCLUSION AND FUTURE WORK}

In this work, numerous three-dimensional CFD simulations were performed to analyse the flow dynamics of a novel nozzle for the filtration unit. The influences of the nozzle shape and dimensions on the entrainment effect were investigated. The following conclusions were drawn from this simulation study: 
(1) The pulse gas formed a wave oscillation downstream of the rectangular nozzle involving the short shock-train in pressure propagation. The attraction force with the first negative pressure region in the pressure propagation was analysed as the main source for the entrainment effect of the pulse gas. The shock wave length increased and the shock wave width was almost constant as the $R_{c h}$ decreased. The number and length of shock waves barely changed with $R_{c w}$ and $R_{d w}$, although the width of shock wave increased with the increasing $R_{c w}$ and $R_{d w}$.

(2) In the rectangular slot nozzle, the dynamic properties of the pulse gas were mostly affected by the nozzle height. The entrainment ratio proportionally increased with the convergent ratio in the narrow $\left(R_{c h}\right)$ and wide $\left(R_{c w}\right)$ directions, as well as the divergent ratio $\left(R_{d w}\right)$ in the wide direction of the nozzle, and was inversely proportional to the pulse pressure. Higher entrainment is desirable to prevent the thermal shock of filter elements working at high temperature because of its mixing effect. The primary mass and secondary gas decreased with the increasing convergent ratio in the narrow $\left(R_{c h}\right)$ and wide $\left(R_{c w}\right)$ directions. The primary mass and secondary gas increased with the increasing divergent ratio $\left(R_{d w}\right)$ in the wide direction of the nozzle.

This simulation model only presented the flow dynamic of pulse cleaning of the nozzle. The diffuser and filter elements of a group pulse system were not covered in this study. However, the strategy of guiding strong and sufficient pulse gas into the diffuser opening, as well as enhancing the mixing effect under a high entrainment condition is an attractive feature of a pulse cleaning system for a filtration unit. The diffuser filter elements should be taken into account in future extensions of this study.

\section{ACKNOWLEDGEMENT}

The study was supported by the National Natural Science Foundation of China (No. U1504217) and by the Korea Institute of Energy Technology Evaluation and Planning (No. 2011201020004C).

\section{REFERENCES}

[1] Chi, H.; Ji, Z.; Sun, D.; Cui, L. (2009). Experimental investigation of dust deposit within ceramic filter medium during filtration-cleaning cycles, Chinese Journal of Chemical Engineering, Vol. 17, No. 2, 219-225, doi:10.1016/S1004-9541(08)60197-4

[2] Qian, Y.; Bi, Y.; Zhang, Q.; Chen, H. (2014). The optimized relationship between jet distance and nozzle diameter of a pulse-jet cartridge filter, Powder Technology, Vol. 266, 191-195, doi:10.1016/j.powtec.2014.06.004

[3] Hata, M.; Furuuchi, M.; Kanaoka, C.; Kurose, R.; Makino, H. (2003). Observation of dust release behavior from ceramic filter elements, Advanced Powder Technology, Vol. 14, No. 6, 719-734, doi: $10.1163 / 15685520360732007$

[4] Chi, H.; Yu, L.; Choi, J.-H.; Ji, Z. (2008). Optimization of nozzle design for pulse cleaning of ceramic filter, Chinese Journal of Chemical Engineering, Vol. 16, No. 2, 306-313, doi:10.1016/S1004-9541(08)60079-8

[5] Schiller, S.; Schmid, H.-J. (2015). Highly efficient filtration of ultrafine dust in baghouse filters using precoat materials, Powder Technology, Vol. 279, 96-105, doi: $\underline{10.1016 /}$ j.powtec.2015.03.048

[6] Alonso-Farinas, B.; Lupion, M.; Rodriguez-Galan, M.; Martinez-Fernandez, J. (2013). New candle prototype for hot gas filtration industrial applications, Fuel, Vol. 114, 120-127, doi:10.1016/j.fuel.2012.12.054

[7] Lo, L.-M.; Chen, D.-R.; Pui, D. Y. H. (2010). Experimental study of pleated fabric cartridges in a pulse-jet cleaned dust collector, Powder Technology, Vol. 197, No. 3, 141-149, doi: $10.1016 /$ j.powtec.2009.09.007 
[8] Choi, J.-H.; Seo, Y.-G.; Chung, J.-W. (2001). Experimental study on the nozzle effect of the pulse cleaning for the ceramic filter candle, Powder Technology, Vol. 114, No. 1-3, 129-135, doi: $10.1016 / \mathrm{S} 0032-5910(00) 00284-9$

[9] Duo, W.; Kirkby, N. F.; Seville, J. P. K.; Clift, R. (1997). Patchy cleaning of rigid gas filters - I. A probabilistic model, Chemical Engineering Science, Vol. 52, No. 1, 141-151, doi:10.1016/ S0009-2509(96)00370-3

[10] Harby, K.; Chiva, S.; Muñoz-Cobo, J. L. (2014). An experimental study on bubble entrainment and flow characteristics of vertical plunging water jets, Experimental Thermal and Fluid Science, Vol. 57, 207-220, doi:10.1016/j.expthermflusci.2014.04.004

[11] Kong, F. S.; Kim, H. D.; Jin, Y.; Setoguchi, T. (2013). Application of Chevron nozzle to a supersonic ejector-diffuser system, Procedia Engineering, Vol. 56, 193-200, doi:10.1016/ j.proeng.2013.03.107

[12] Stocklmayer, C.; Hoflinger, W. (1998). Simulation of long-term behaviour of regenerateable dust filters, Filtration \& Separation, Vol. 35, No. 4, 373-377, doi:10.1016/S0015-1882(97)87418-6

[13] Stocklmayer, C.; Hoflinger, W. (1998). Simulation of the regeneration of dust filters, Mathematics and Computers in Simulation, Vol. 46, No. 5-6, 601-609, doi:10.1016/S03784754(98)00089-5

[14] Zhang, X.; Chen, H.; Ji, Z.; Wang, J. (2009). Characterization of flow field in the exit of pulse jet nozzle for high-temperature filtrating, Chinese Journal of Mechanical Engineering, Vol. 45, No. $10,96-100$

[15] Mohammed, R. H. (2013). A simplified method for modeling of round and square ceiling diffusers, Energy and Buildings, Vol. 64, 473-482, doi:10.1016/j.enbuild.2013.05.021

[16] Schulz, K.; Durst, M. (1994). Advantages of an integrated system for hot gas filtration using rigid ceramic elements, Filtration \& Separation, Vol. 31, No. 1, 25-28, doi:10.1016/00151882(94)80227-0

[17] Wang, J.-F.; Piechna, J.; Müller, N. (2012). A novel design of composite water turbine using CFD, Journal of Hydrodynamics, Ser. B, Vol. 24, No. 1, 11-16, doi:10.1016/S10016058(11)60213-8

[18] Launder, B. E.; Spalding, D. B. (1972). Mathematical Models of Turbulence, Academic Press, London 